\title{
Fortbilden mit conrad: „Best of RÖKO 2020“ und Aufzeichnungen der Kardiodiagnostiktage verfügbar
}

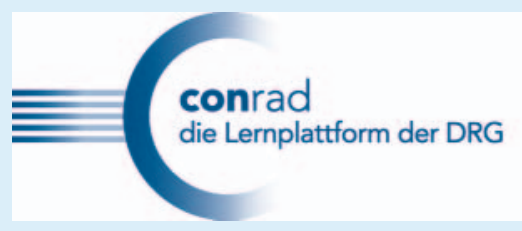

Auf conrad, der interaktiven Lernplattform der Deutschen Röntgengesellschaft, finden Interessierte unter dem Titel „Best of RÖKO 2020“ einen Kurs, mit den am besten bewerteten Sitzungen des vergangenen Röntgenkongresses. Viele Teilnehmende haben angeregt, die auf conrad angebotenen Aufzeichnungen für die radiologische Weiter- und Fortbildung für Radiologinnen und Radiologen längerfristig verfügbar zu machen. Diesem Wunsch sind wir gerne gefolgt. Die zuständige Ärztekammer hat die Kurse anerkannt, sodass die Lernenden CME-Punkte für die Bearbeitung erhalten können.

Die Inhalte des „Best of RÖKO 2020“ sind weit gefasst: So stehen neben der Gastrointestinal- und Abdominaldiagnostik auch die Thoraxradiologie, die Notfalldiagnostik und die Muskuloskelettale Radiologie auf der Lernplattform zur Verfügung. Ein besonderes Highlight sind die Sitzungen aus dem Bereich des Programms „Fit für den Facharzt“ (FFF). Hier finden Sie zudem Vorträge

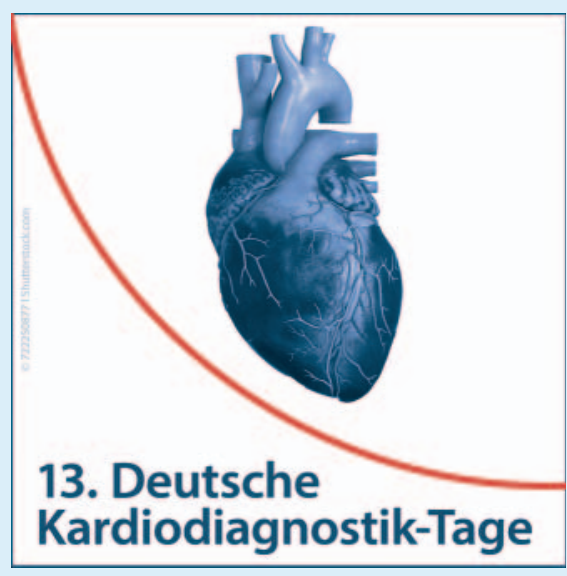

zu den Themen Mammadiagnostik, Uroradiologie und Herzbildgebung.

Ein weiteres Highlight auf conrad sind die Aufzeichnungen der Q-Kurse zur Herzbildgebung im Rahmen der 13. Deutschen Kardiodiagnostiktage, die im Februar dieses Jahres digital stattgefunden haben. Neben den Vorträgen der Q1- und Q2-Kurse HerzCT und Herz-MRT finden Interessierte drei thematisch passende Fallsammlungen auf der Lernplattform: die „Fallsammlung HerzCT der AG Herz- und Gefäßdiagnostik“, die „Fallsammlung Herz-MR der AG Herz- und Gefäßdiagnostik“ sowie die Fallsammlung „Kongenitale Herzfehler im Kindes- und Erwachsenenalter (EMAH)“.
Bitte beachten Sie, dass Sie für eine persönliche Zertifizierung in der Herz- und Gefäßdiagnostik die Teilnahme an den jeweils erforderlichen Q-Kursen in Form von Präsenzveranstaltungen oder Live-Webinaren nachweisen müssen, die Bearbeitung der aufgezeichneten Vorträge auf conrad ist nicht ausreichend. Die bearbeiteten Fälle aus den oben genannten Fallsammlungen können Sie für die Zertifizierung in vollem Umfang als Nachweis durchgeführter Untersuchungen anrechnen lassen.

Ein wichtiges Ziel der Deutschen Röntgengesellschaft ist es, Fachwissen zeit- und ortsunabhängig verfügbar zu machen und das fachspezifische Lernen mit hochwertigen Angeboten zu bereichern. Der „Best of RÖKO 2020“ und die Aufzeichnungen der Q-Kurse zur Herzbildgebung auf conrad sind weitere Schritte in diese Richtung.

Haben Sie Fragen oder Wünsche zu den Kursen auf conrad? Dann kontaktieren Sie in der DRG-Geschäftsstelle gerne:

Olaf Goldschmidt

Tel: 030-916 070-47

Mail: goldschmidt@drg.de 